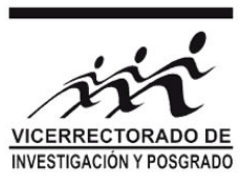

\title{
Análisis de un Sistema de Referencia Inercial
}

\author{
Oscar Monroy ${ }^{*} 1$ \\ ${ }^{1}$ Universidad Nacional Mayor de San Marcos, Ap. Postal 14-0149, Lima, Perú
}

Recibido 03 abril 2018 - Aceptado 28 julio 2018

\begin{abstract}
Se desarrolla la idea del universo abierto con el propósito de descifrar el misterio del sistema de referencia inercial. Se deduce que una primera aproximación a un sistema de referencia inercial sería el centro de una esfera tetradimensional. La interpretación objetiva de los experimentos de la inversión especular y del espín del electrón requiere considerar un eje de rotación situado en una dimensión espacial extra, asociado a cada sistema natural autoorganizado y autónomo del espacio tridimensional. Además, se deduce que el universo observable podría concebirse como un sistema dual, con dos superficies complementarias opuestas simétricas las cuales conformarían una sola entidad. Si una de las superficies corresponde a la mente consciente del observador, la otra superficie debe corresponder a la mente inconsciente del observador. Reinterpretando el concepto de hiperesfera e introduciendo el concepto de dimensiones espaciales complementarias se construyen diagramas para mostrar que el universo observable es un sistema abierto y dinámico.

Palabras claves: Sistema de referencia inercial, universo abierto, esfera tetradimensional, inversión especular, dimensiones espaciales complementarias..
\end{abstract}

\section{Analysis of an Inertial Reference System}

The idea of the open universe is developed in with the purpose of deciphering the mystery of the inertial reference system. It follows that a first approximation to an inertial reference system would be the center of a four-dimensional sphere. The objective interpretation of the experiments of the mirror inversion and the spin of the electron requires considering an axis of rotation located in an extra spatial dimension, associated with each natural self-organized and autonomous system of three-dimensional space. In addition, it is deduced that the observable universe could be conceived as a dual system, with two opposite symmetrical complementary surfaces which would form a single entity. If one of the surfaces corresponds to the conscious mind of the observer, the other surface must correspond to the unconscious mind of the observer. Reinterpreting the hypersphere concept and introducing the concept of complementary spatial dimensions, diagrams are constructed to show that the observable universe is an open and dynamic system.

Keywords: Inertial reference system, open universe, four-dimensional sphere, mirror reversion, complementary spatial dimensions..

\section{Introducción}

Un cuerpo en el cual no actúan fuerzas externas netas se llama sistema de referencia inercial. Las leyes de Newton de la mecánica son aplicables en esta clase de sistema de referencia. El concepto de sistema de referencia inercial es una consecuencia del principio de inercia de Newton, el cual afirma que un cuerpo permanecerá en reposo o tendrá movimiento rectilíneo uniforme si no hay fuerzas externas que actúen sobre él. Si admitimos que el universo observable es un sistema cerrado y dinámico, no existirá ningún cuerpo material al cual se le pueda aplicar el principio de inercia. Por esta razón un sistema de referencia inercial es

\footnotetext{
*oscarmonroycardenas@hotmail.com
}

considerado una ficción [1], [2], [3]. ¿Habrá alguna manera de hallar un sistema inercial?

La Tierra no es un sistema de referencia inercial porque gira alrededor de su eje, y a su vez alrededor del Sol. El Sol no es un sistema de referencia inercial porque gira alrededor del centro de la Vía Láctea. Las galaxias y los cúmulos de galaxias tampoco son sistemas de referencia inerciales porque giran las unas alrededor de las otras. Entonces nos hallamos en un universo dinámico donde todos los objetos giran. No existe ningún objeto material en el espacio tridimensional el cual pueda ser considerado un sistema de referencia inercial. Por tanto, ningún objeto localizado en el espacio tridimensional puede ser considerado un sistema 
de referencia inercial.

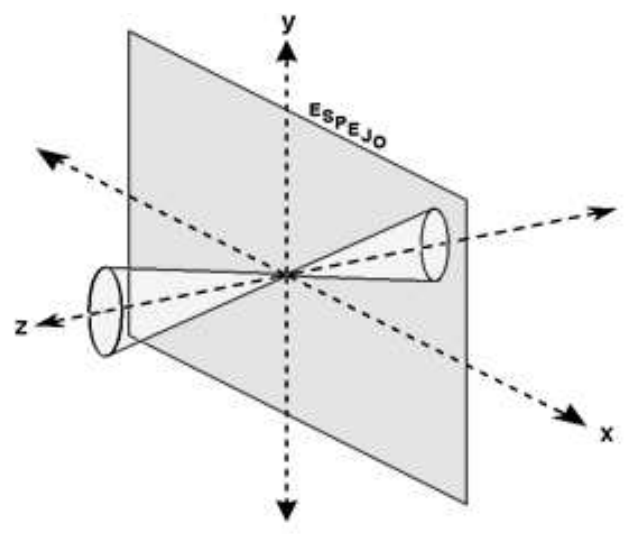

Figura 1: No hay distinción física entre espacio real y espacio virtual. Ambos espacios coexisten.

En un artículo anterior [2] se mostró que la relación de incertidumbre energía-tiempo implica la posibilidad de que existan agujeros en el espacio tridimensional a través de los cuales ingrese energía para la creación de pares partícula/antipartícula virtuales. Por consiguiente, podría admitirse que el universo observable sea un sistema abierto hacia un espacio de dimensión superior. Entonces surge la pregunta: ¿existirá un sistema de referencia inercial en un espacio de dimensión mayor? Para responder esta pregunta se puede partir del principio cosmológico convencional, el cual dice que el universo a gran escala exhibe simetría esférica [3]. Esta peculiar visión nos sugiere que hay algo especial respecto a nuestra posición en el universo. Pero, esta visión no es respecto a nuestro cuerpo material sino respecto a nuestra mente [4].

¿Qué es la mente? Sólo tiene sentido concebir el concepto de la mente en el contexto de los sistemas autoorganizados, autónomos y con funcionamiento inteligente. Carece de sentido definir la mente a nivel de los elementos constituyentes de dichos sistemas. Por consiguiente, la mente tiene la propiedad de ser una entidad holística.

En general, se puede afirmar que la mente sería la información organizada que recibe un sistema autoorganizado para que sea procesada por los elementos constituyentes de éste. En los sistemas biológicos, y en particular en el cuerpo humano, la información organizada se procesaría en el cerebro desde sus distintos órganos.

La mente humana actuaría sobre la materia a través del fenómeno de volición. Según la filosofía del realismo volitivo, se concibe la consciencia como un esfuerzo o acción originario al que se opone la realidad exterior en forma de obstáculo o resistencia [5]. Admitiendo este pensamiento, y que existen los estados de consciencia/inconsciencia asociados a la mente del observador, entonces la mente de un sistema natural puede concebirse como una fuerza que determina los procesos físicos.
Por otro lado, el hecho de que nuestra visión del universo a gran escala se describa con simetría esférica, significaría que la mente humana lee esta propiedad del universo desde un lugar privilegiado. Por consiguiente, este hecho nos califica como observadores fundamentales del universo. Además, dicho lugar de visión privilegiado (que puede denominarse foco de lectura o de visión de la mente humana) debe hallarse en un espacio de dimensión mayor, de lo contrario nuestra visión del universo a gran escala sería diferente desde posiciones y direcciones diferentes respecto a la ubicación de nuestro cuerpo material. Así, desde esta perspectiva la mente humana puede trascender los límites terrestres.

Introducir en la cosmología el concepto de la mente como una fuerza que influye sobre los sistemas naturales constituye una nueva ciencia. Por consiguiente, la búsqueda de un sistema de referencia inercial implica investigar el lugar especial en un espacio de dimensión superior donde se hallaría el foco de lectura de la mente humana. Además, sabemos que nuestra línea de visión de los objetos del universo es la línea de propagación de la luz, y está estrechamente vinculada al foco de lectura de la mente. Toda nuestra información de los objetos lejanos o cercanos del mundo físico que llega a nuestra consciencia depende principalmente de la propagación de la luz.

\section{Consideraciones geométricas}

Para comprender de la manera más simple posible lo que implicarían los razonamientos anteriores, imaginemos que el universo es una esfera tridimensional y que todos los objetos que contiene se encuentran distribuidos en su superficie. Entonces para que la mente de un observador pueda leer el universo con simetría esférica en cualquier lugar de la superficie esférica y en cualquier dirección, los rayos luminosos procedentes de los objetos del universo deberán converger en el centro de la esfera. Por consiguiente, la única posibilidad de que cada observador de este universo tenga la misma visión del universo sería que el foco de visión de su mente sea el centro de dicha esfera.

Un ser poco inteligente, cuyo cuerpo material se encuentre en la superficie esférica, tendrá la sensación psicológica de que el lugar donde se encuentra es el centro del universo y que todo gira alrededor de él. Otro ser un poco más inteligente deducirá que su visión es engañosa; que su lugar de referencia no es el centro del universo y tratará de hallar un sistema de referencia inercial en la materia. Pero un ser mucho más inteligente se percataría que ningún cuerpo material puede considerarse un sistema de referencia inercial. Luego, deduciría que la clave podría estar en la mente del observador, siempre que el foco de la visión sea un lugar especial que trasciende los límites terrestres, intentando resolver así el misterio de su peculiar visión. 


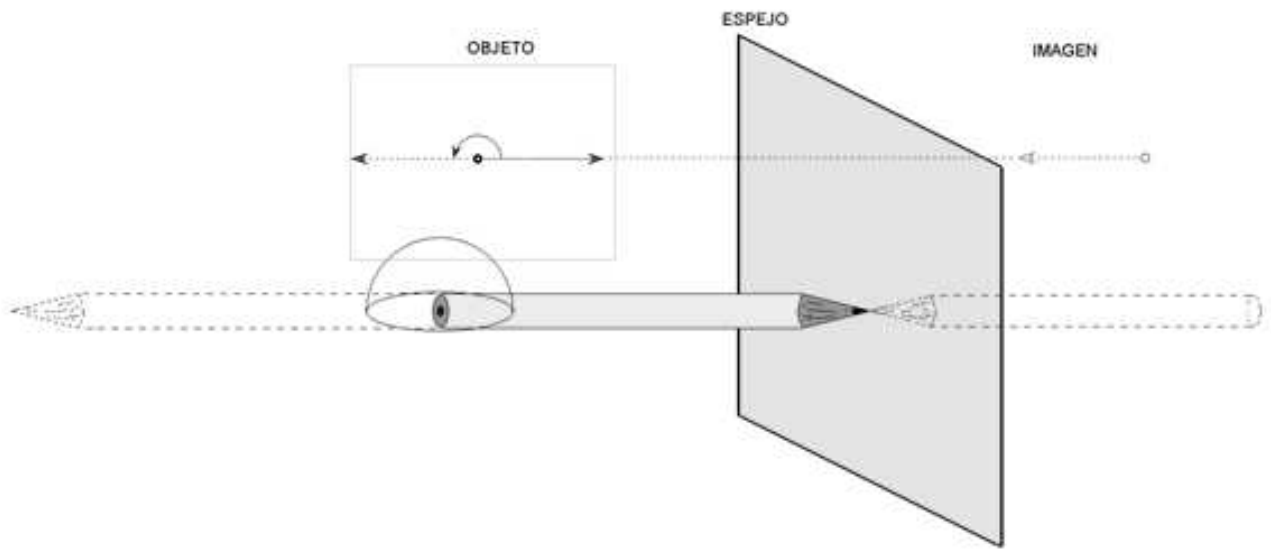

Figura 2: La inversión especular de un objeto bidimensional (la flecha) se describe con el ángulo plano ( $\pi$ radianes) y requiere de un eje de rotación perpendicular al plano. En cambio la inversión especular de un objeto tridimensional (el lápiz) se describe con el ángulo sólido ( $2 \pi$ estereoradianes) y requiere de un eje de rotación situado en una dimensión extra.

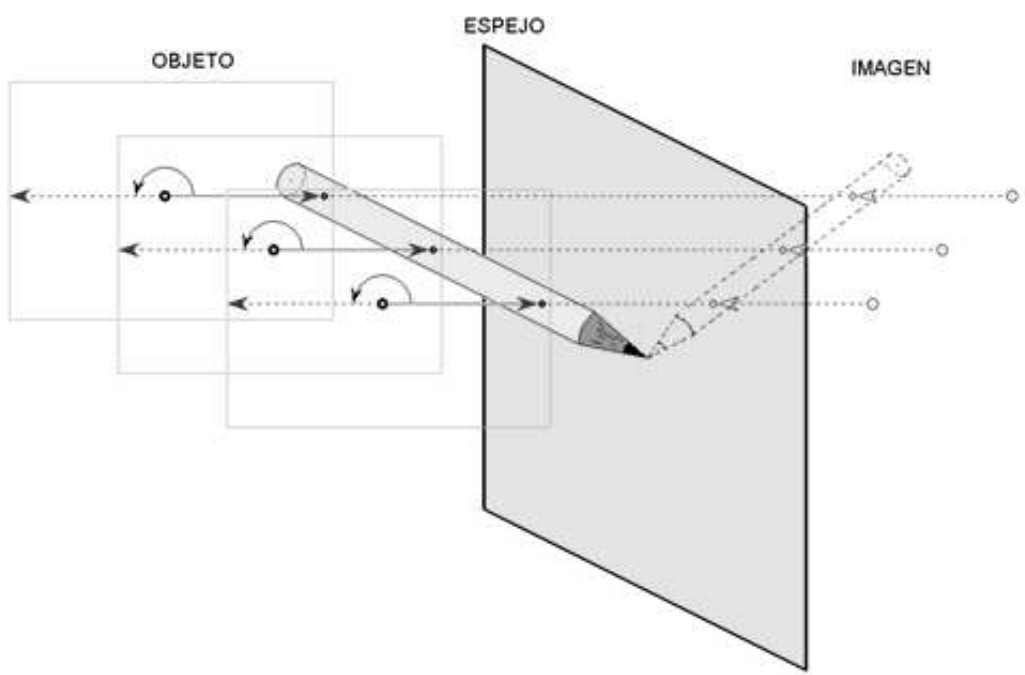

Figura 3: La superficie opuesta de un objeto tridimensional (el lápiz) es su imagen en el espejo, siempre que las normales (flechas) desde cada punto del objeto y desde cada punto de su imagen respecto al espejo sean opuestas entre si.

En la perspectiva del universo que se ha descrito, la superficie esférica (el espacio bidimensional) correspondería al mundo subjetivo de la percepción sensorial y el espacio interior de la esfera (es decir, el espacio tridimensional) correspondería a un mundo objetivo. Por esta razón se puede pensar que el foco de visión de la mente del observador se encuentra ubicado en el centro de la esfera. Por tanto, se deduce que el mundo subjetivo situado sobre la superficie esférica se complementa con el mundo objetivo localizado en el interior de la esfera conformando así un universo aislado estático. Pero para que sea dinámico deberá existir un eje de rotación fijo en una dimensión extra a la esfera. ¿Se podrán extrapolar estas ideas hacia una esfera tetradimensional para empezar a investigar la realidad objetiva de nuestro universo?

También, desde el punto de vista geométrico es posible extender nuestra noción preconcebida de esfera a espacios de dimensiones mayores a tres (Ilamada hiperesfera), aunque nunca se haya podido imaginar su aspecto. Sin embargo, en la Física las extrapolaciones se justifican sobre la base de la evidencia experimental. Es un hecho comprobado por la experiencia de la vida cotidiana es que, en condiciones normales, somos conscientes del aspecto que tiene la superficie cerrada que envuelve a un cuerpo tridimensional (realidad subjetiva) pero somos inconscientes de lo que está ocurriendo en su interior (realidad objetiva). Este razonamiento puede aplicarse a los sistemas naturales autónomos como el cuerpo humano, la Tierra y 
por consiguiente al propio universo. Se percibe el universo superficialmente (en tres dimensiones) de la forma que es (realidad subjetiva) pero no se percibe directamente su interior (en cuatro dimensiones) aparentemente oculto (realidad objetiva). En este sentido la realidad objetiva es independiente de la consciencia humana.

Es claro que todo cuerpo material ocupa un lugar en el espacio tridimensional. Extrapolando el razonamiento del párrafo anterior se puede pensar que los objetos materiales se encuentran distribuidos sobre una superficie tridimensional y que detrás de ella existe un mundo claro y objetivo. También, se puede pensar que este mundo trascendente es un espacio de cuatro dimensiones donde habría una entidad la cual sostiene y dirige a los cuerpos materiales que se localizan en la superficie tridimensional. Tal entidad puede identificarse como información organizada. Por consiguiente, esto implica que la mente sería información organizada implantada en los sistemas naturales para que estos funcionen con inteligencia.

Por otro lado, en la cosmología convencional el problema del origen del universo significa estudiar el origen del tiempo [4]. Se acepta la idea de que el universo nació junto con el tiempo. Pero el funcionamiento del universo induce a pensar que es también un sistema natural autoorganizado, autónomo y con funcionamiento inteligente, es decir que tiene mente. Además, se distinguen varias direcciones del tiempo como: el tiempo cosmológico, el tiempo termodinámico y el tiempo psicológico [4]. Como estas direcciones se refieren a la evolución de los sistemas naturales, habría una relación estrecha entre la naturaleza del tiempo y la mente del universo.

Para desarrollar la idea del universo abierto, en un intento por hallar un sistema de referencia inercial, se utiliza el criterio de que todo sistema natural autoorganizado, autónomo y con funcionamiento inteligente posee una abertura o agujero tridimensional a través del cual se ejecuta la información organizada para su funcionamiento. Entonces, desde el punto de vista geométrico, es conveniente establecer una conexión entre el espacio tridimensional y el espacio tetradimensional considerando un eje de rotación situado en una dimensión espacial extra, a partir del cual se sustente la dinámica esencial del universo observable.

A continuación se consideran dos experimentos con el propósito de mostrar que el sistema de referencia objetivo no es un objeto localizado en el espacio físico sino el foco de visión de la mente humana, y que ésta influiría sobre la materia desde un espacio de dimensión mayor. Se toma como punto de partida la idea de que el universo es un sistema abierto, dinámico, con simetrías esférica y especular. Finalmente se explican estas propiedades mediante diagramas construidos a partir de nuestra noción preconcebida de esfera.

\section{El universo como sistema abierto}

Se propone que si el universo observable es un sistema abierto entonces todo sistema natural autoorganizado, autónomo y con funcionamiento inteligente (es decir, con mente) posee una abertura o agujero a través del cual es revitalizado, posiblemente desde un espacio de dimensión mayor. Además, para que el universo observable sea dinámico se requiere que exista un eje de rotación situado en una dimensión espacial extra a las tres dimensiones espaciales que percibimos, el cual esté conectado de algún modo a los ejes espaciales que percibimos. Pero, ¿qué experimentos pueden justificar la idea del universo abierto y dinámico?

\subsection{El experimento de la inversión especular}

El universo observable tendría la propiedad de ser un sistema dual constituido por dos superficies tridimensionales complementarias opuestas con simetría especular, las cuales conformarían una sola entidad. Si una de las superficies corresponde a la mente consciente del observador, la otra superficie debe corresponder a la mente inconsciente del observador. Esta interpretación puede explicarse y comprobarse mediante el experimento cotidiano del espejo plano.

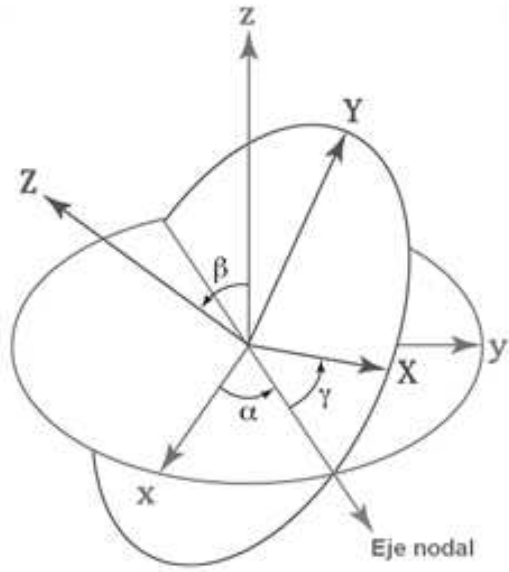

Figura 4: Ángulos de Euler $(\alpha, \beta$ y $\gamma)$. Estos ángulos indican rotaciones en el espacio físico tridimensional alrededor de tres ejes. Se utilizan en la teoría cuántica del momento angular para construir el operador de rotación.

Existe una profunda significación filosófica en las imágenes especulares [4], [6]. Alúmbrese un espejo plano con una linterna en la oscuridad. Se observa que la luz atraviesa sin dificultad la superficie del espejo enfocando cualquier objeto en el espacio al otro lado del espejo; al cual llamaremos espacio virtual. Por consiguiente, la superficie del espejo se comporta como si fuese una ventana que 
permite el paso de la luz hacia el espacio virtual. Y viceversa, la luz procedente del espacio virtual también ilumina simultáneamente el espacio donde somos conscientes; al cual llamaremos espacio real. Esto significaría que ambos espacios coexisten conformando las dos caras del espacio tridimensional.

El principio de conservación de la Paridad implica que nunca podemos estar completamente seguros si estamos observando un fenómeno físico en el espacio real o en el espacio virtual. En el espacio tridimensional no se pueden distinguir sus dos caras. Pero si observáramos los objetos desde el espacio tetradimensional, se podría comprender el anverso y el reverso de un objeto tridimensional, y seríamos conscientes de que conforman una sola entidad. Lo que ocurre es que nuestra mente está habituada a observar los objetos del espacio tridimensional de modo unilateral, adquiriendo la profunda sensación subjetiva de que el espacio virtual es solo ilusión óptica.

Del análisis anterior, surge una aparente paradoja: si los espacios real y virtual coexisten, y cada persona junto con su imagen observan simultáneamente los fenómenos físicos desde ambos lados del espacio tridimensional, entonces no habría una sola posición de observación, sino dos. Sin embargo, es posible observar simultáneamente los objetos en el espacio real y en el espacio virtual. Por ejemplo, curiosamente el cono en la posición mostrada en la figura 1 , puede verse simultáneamente en ambos espacios. Entonces, ¿desde qué posición estamos observando realmente los objetos del espacio tridimensional? La única posibilidad es que nuestra posición de observación (es decir, el foco de lectura de la mente) no se encuentre en este mundo, sino en un lugar especial del espacio tetradimensional. Este sería el sistema de referencia de la mente del observador.

Si el espacio virtual fuese ilusorio, entonces no existiría la sombra proyectada por un objeto. Para aclarar esto, se puede considerar otro experimento. Se sabe que la sombra que se proyecta sobre una superficie opaca es parte inseparable de todos los cuerpos materiales. Si se pule indefinidamente una superficie rígida opaca, sobre la cual se proyecta la sombra de un objeto material, se convertirá finalmente en un espejo ideal y se verá que la sombra desaparece. ¿Dónde se encontrará la sombra?, ¿ya no existe? Sí existe; lo que ocurre es que se ha proyectado sobre el espacio virtual. Pero a su vez, la imagen especular también se proyectará sobre el espacio real. Por consiguiente, los espacios real y virtual coexisten conformando una sola entidad.

Un objeto material o su imagen especular no constituyen un sistema de referencia objetivo, sino el sistema de referencia de la mente del observador. El origen de este sistema de referencia (el foco de lectura de la mente) sería el punto de concurrencia de las líneas de visión humana (rayos de luz), y se localizaría en un lugar común: el centro de una inimaginable esfera tetradimensional (al menos en primera aproximación). Por tanto, el universo debe ser un sistema abierto (con aberturas existentes en los sistemas naturales con mente, como nosotros) hacia este mundo trascendente.

Si estos argumentos se aplican al experimento del espejo, significará que los objetos del espacio tridimensional, que se proyectan a través del espejo, tendrán su centro de curvatura en el centro de la esfera tetradimensional. Pero, hay que percatarnos que la proyección de un objeto a través de un espejo significa una inversión de la dirección perpendicular (la normal) al espejo correspondiente a cada punto del objeto y se llama inversión especular.

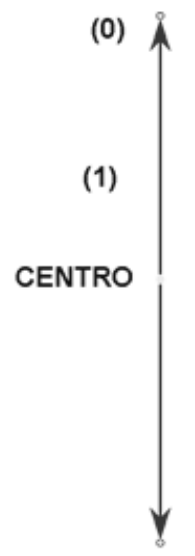

Figura 5: Diagrama elemental para explicar la idea del universo abierto. Las dimensiones complementarias son 0 y 1 . La doble flecha proveniente del centro indica la dualidad intrínseca asociada a los puntos físicos objeto e imagen (extremos) los cuales conforman una sola entidad.

La inversión especular de un objeto bidimensional a través de un espejo unidimensional equivale a una rotación de $\pi$ rad alrededor de un eje inercial situado en una dimensión extra al espacio bidimensional. Es claro que el retorno del objeto a su orientación inicial equivale a una vuelta, que habitualmente se percibe, y la medida del ángulo plano (bidimensional) correspondiente a una vuelta es $2 \pi \mathrm{rad}$.

Análogamente, la inversión especular de un objeto tridimensional a través de un espejo bidimensional equivale a media vuelta por el espacio tetradimensional. Puesto que la medida de un ángulo de rotación tridimensional es el ángulo sólido, entonces la inversión especular equivale a una rotación $2 \pi$ alrededor de un eje inercial situado en una dimensión extra al espacio tridimensional, el cual no se percibe. El retorno del objeto tridimensional a su orientación inicial equivale a una vuelta por el espacio tetradimensional, y la medida del ángulo sólido correspondiente es igual a $4 \pi$. Y así, extrapolando, la inversión especular de un objeto tetradimensional a través de un espejo tridimensional equivale a una rotación (media vuelta) $3 \pi$ 
por el espacio pentadimensional. Entonces el retorno del objeto a su orientación inicial equivale a una rotación $6 \pi$.

En general, la inversión especular de un objeto de dimensión $\mathrm{n}$ a través de un espejo de dimensión $\mathrm{n}-1$ equivale a una rotación $(n-1) \pi$ por el espacio de dimensión $n$ +1 , (donde $n=1,2,3, \ldots$ ). Por consiguiente, el retorno del objeto a su orientación inicial implica una rotación 2 (n - 1) $\pi$. Por tanto, en cada espacio de dimensión $n+1$ podemos elegir un eje de rotación inercial, y por consiguiente un sistema de referencia inercial cuyo origen sería el centro de una hiperesfera de dimensión $n+1$.

En la figura 2 se explica la inversión especular de un objeto tridimensional (el lápiz). Un espejo invierte la dirección perpendicular a él. La inversión especular de un objeto bidimensional plano (la flecha perpendicular al espejo) se describe mediante el ángulo plano ( $\pi$ radianes) y se requiere considerar un eje de rotación perpendicular al plano. En cambio la inversión especular de un objeto tridimensional (el lápiz perpendicular al espejo) se describe mediante el ángulo sólido ( $2 \pi$ estereoradianes) y se requiere considerar un eje de rotación perpendicular al espacio tridimensional, el cual no se percibe. Puesto que nuestra percepción sensorial común no permite comprobar directamente la existencia de este cuarto eje, se requeriría otro tipo de percepción sensorial. Además, del análisis del experimento de la inversión especular se deduce que la superficie opuesta de un objeto bidimensional es su imagen en un espejo unidimensional, siempre que las normales al espejo desde cada punto del objeto y desde cada punto de su imagen sean opuestas entre si.

Análogamente, la superficie opuesta de un objeto tridimensional es su imagen en un espejo bidimensional, siempre que las normales al espejo desde cada punto del objeto y desde cada punto de su imagen son opuestas entre si. Por ejemplo, en la figura 3 se muestra un lápiz en una posición arbitraria respecto al espejo. La normal desde cada punto del lápiz al espejo es opuesta a la normal desde cada punto de su imagen al espejo.

Las observaciones anteriores se pueden generalizar planteando el siguiente postulado: la superficie opuesta de un objeto de dimensión $n$ es su imagen en un espejo de dimensión $n-1$, siempre que las normales al espejo desde cada punto del objeto y desde cada punto de su imagen sean opuestas entre si. Por tanto, se llega a la conclusión de que el universo observable es un sistema dual: tiene dos superficies tridimensionales complementarias opuestas con simetría especular las cuales conforman una sola entidad.

\subsection{El experimento del espín del electrón}

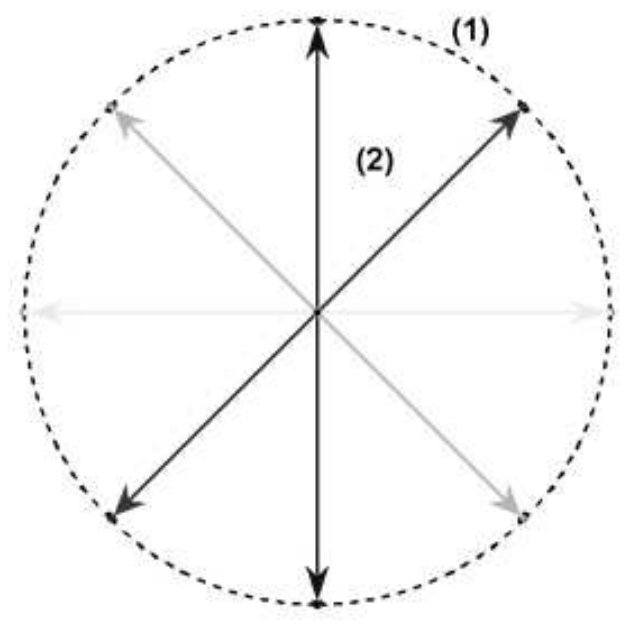

Figura 6: Diagrama para explicar la idea del universo abierto en la dimensión uno (1). Se indica la dimensión complementaria dos (2). El eje de rotación inercial pasa por el centro del circulo, es perpendicular al papel, y se sitúa en la dimensión tres (3).

En la teoría cuántica del momento angular la explicación de la rotación $4 \pi$ que requiere el electrón (y en general todas las partículas de espín 1/2) para poder retornar a su orientación inicial es insuficiente, porque se basa en el criterio de los ángulos de Euler los cuales indican la orientación de un sólido en el propio espacio tridimensional, como se muestra en la figura 4. El experimento del espín del electrón puede interpretarse como una evidencia de la influencia de la mente en la materia. Se observa que el espín asociado a un electrón siempre se orientará paralelamente a un eje del espacio tridimensional que el experimentador elija como referencia. La descripción del estado de espín del electrón que se realiza en la mecánica cuántica no es respecto al espacio físico, sino mediante un artificio matemático llamado espacio de espinores. Cada elemento de este espacio se llama espinor, el cual caracteriza el estado del electrón y se representa por la matriz columna de dos componentes:

$$
\Psi=\left(\begin{array}{c}
\Psi_{+} \\
\Psi_{-}
\end{array}\right)
$$

donde $\Psi_{+}$y $\Psi_{-}$son funciones de onda dependientes de las tres coordenadas espaciales, con subíndices discretos \pm , los cuales corresponden a las dos posibles orientaciones del espín del electrón.

La matriz de rotación para partículas de espín $1 / 2$ está dada por la expresión: 


$$
R^{\left(\frac{1}{2}\right)}(\alpha \beta \gamma)=\left(\begin{array}{cc}
e^{\frac{-i \alpha}{2}} \cos \left(\frac{\beta}{2}\right) e^{\frac{-i \gamma}{2}} & e^{\frac{-i \alpha}{2}} \sin \left(\frac{\beta}{2}\right) e^{\frac{-i \gamma}{2}} \\
e^{\frac{i \alpha}{2}} \sin \left(\frac{\beta}{2}\right) e^{\frac{-i \gamma}{2}} & -e^{\frac{i \alpha}{2}} \cos \left(\frac{\beta}{2}\right) e^{\frac{i \gamma}{2}}
\end{array}\right)
$$

donde $\alpha, \beta$ y $\gamma$ son los ángulos de Euler.

Por otro lado, una rotación del espinor se puede representar por:

$$
\left(\begin{array}{c}
\Psi_{+}^{\prime} \\
\Psi_{-}^{\prime}
\end{array}\right)=R^{\left(\frac{1}{2}\right)}(\alpha \beta \gamma)\left(\begin{array}{c}
\Psi_{+} \\
\Psi_{-}
\end{array}\right)
$$

Para una rotación alrededor del eje $\mathrm{z}: \beta=0, \gamma=0, \mathrm{y}$ la Ec.(2) se reduce a:

$$
R^{\left(\frac{1}{2}\right)}(\alpha)=\left(\begin{array}{cc}
e^{\frac{-i \alpha}{2}} & 0 \\
0 & e^{\frac{i \alpha}{2}}
\end{array}\right)
$$

Reemplazando (4) en (3) se tiene:

$$
\begin{gathered}
\left(\begin{array}{c}
\Psi_{+}^{\prime} \\
\Psi_{-}^{\prime}
\end{array}\right)=\left(\begin{array}{cc}
e^{\frac{-i \alpha}{2}} & 0 \\
0 & e^{\frac{i \alpha}{2}}
\end{array}\right)\left(\begin{array}{l}
\Psi_{+} \\
\Psi_{-}
\end{array}\right) \\
\left(\begin{array}{c}
\Psi_{+}^{\prime} \\
\Psi_{-}^{\prime}
\end{array}\right)=\left(\begin{array}{c}
e^{\frac{-i \alpha}{2}} \Psi_{+} \\
e^{\frac{i \alpha}{2}} \Psi_{-}
\end{array}\right)
\end{gathered}
$$

Para una rotación $\alpha=2 \pi$ se obtiene:

$$
\left(\begin{array}{c}
\Psi_{+}^{\prime} \\
\Psi_{-}^{\prime}
\end{array}\right)=\left(\begin{array}{c}
e^{\frac{-i(2 \pi)}{2}} \Psi_{+} \\
e^{\frac{i(2 \pi)}{2}} \Psi_{-}
\end{array}\right)=-\left(\begin{array}{c}
\Psi_{+} \\
\Psi_{-}
\end{array}\right)
$$

Para una rotación $\alpha=4 \pi$ se obtiene:

$$
\left(\begin{array}{c}
\Psi_{+}^{\prime} \\
\Psi_{-}^{\prime}
\end{array}\right)=\left(\begin{array}{c}
e^{\frac{-i(4 \pi)}{2}} \Psi_{+} \\
e^{\frac{i(4 \pi)}{2}} \Psi_{-}
\end{array}\right)=-\left(\begin{array}{c}
\Psi_{+} \\
\Psi_{-}
\end{array}\right)
$$

Los resultados (6) y (7) significan que el electrón (y en general las partículas de espín $1 / 2$ ) requieren aparentemente de dos vueltas para retornar a su orientación inicial. Este resultado es extraño y misterioso si el eje de rotación se localiza en el interior del espacio tridimensional. Pero, si el eje de rotación se localiza en el espacio tetradimensional resulta natural que el electrón retorne a su orientación inicial, porque una vuelta en un espacio de cuatro dimensiones equivale a $4 \pi$. Por consiguiente, el experimento del espín del electrón es otra prueba de que el universo tridimensional es un sistema abierto y que el sistema de referencia adecuado para describir la rotación $4 \pi$ del electrón es el centro de una esfera tetradimensional.

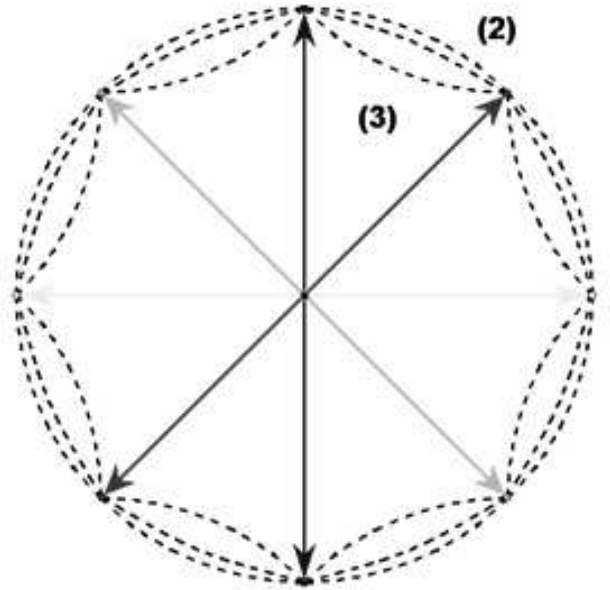

Figura 7: Diagrama para explicar la idea del universo abierto en la dimensión dos (2). Se indica la dimensión complementaria tres (3). El eje de rotación inercial pasa por el centro de la esfera, se localiza en la dimensión cuatro (4), pero no se percibe.

\section{Dimensiones espaciales complementa- rias}

El problema de la dimensionalidad espacial ha sido enfocado por diversos autores de textos de divulgación científica quienes sostienen implícitamente la tesis de que el universo es un sistema cerrado [7], [8], [9], [10]. Sin embargo, la propuesta del universo abierto plantea la posibilidad de hallar un sistema de referencia inercial (al menos en primera aproximación) y además, relacionar las dimensiones espaciales. Esto permitiría investigar la acción de influencias externas procedentes desde un espacio de dimensión superior sobre un espacio de dimensión inferior para tratar de comprender la dinámica del universo observable. Se partirá de la idea de que el universo es un sistema abierto, dinámico, con simetrías esférica y especular. También, se hacen algunas consideraciones geométricas relacionadas con el concepto general de superficie esférica, lo cual es conveniente aclarar ahora.

Una superficie esférica es el conjunto de puntos de un espacio de dimensión cualquiera $(0,1,2,3, \ldots)$ que se encuentran a la misma distancia de un punto llamado centro de curvatura. Una superficie esférica en un espacio de dimensión cero tiene su centro de curvatura en un punto del espacio de dimensión uno. Una superficie esférica en un espacio de dimensión uno tiene su centro de curvatura en un punto del espacio de dimensión dos. Una superficie en un espacio de dimensión dos tiene su centro de curvatura en un punto del espacio de dimensión tres [8]. Entonces una superficie esférica en un espacio de dimen- 
sión tres tendrá su centro de curvatura en un punto del espacio de dimensión cuatro: el centro de la esfera tetradimensional. Podemos seguir razonando de esta manera por inducción sin límite. Las superficies y espacios que se relacionan de esta manera con dimensiones espaciales consecutivas conforman una hiperesfera. Si la hiperesfera es de dimensión $\mathrm{n}$, su superficie será de dimensión $n^{\smile} 1$, (donde $\mathrm{n}=1,2,3, \ldots$ ). Nótese que esta proposición constituye un postulado geométrico, el cual afirma que para definir una hiperesfera las dimensiones espaciales deben complementarse de modo consecutivo. Es de interés aplicar este postulado para relacionar la superficie tridimensional que percibimos (es decir, el espacio tridimensional) con su complementario, el espacio tetradimensional.

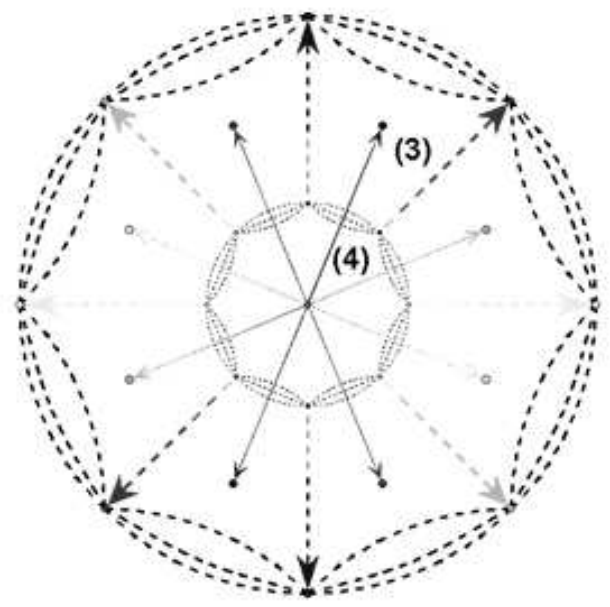

Figura 8: Diagrama para explicar la idea del universo abierto en la dimensión tres (3). Los sectores cónicos corresponden a la superficie de la esfera tetradimensional (4). Las flechas duales indicarían ejes de rotación tetradimensionales que estarían conectados a través de las aberturas a los sistemas, y pueden considerarse inerciales para cada uno de ellos. El eje de rotación inercial para el universo observable debe pasar por el centro de la esfera tetradimensional, se localiza en la dimensión cinco (5) y sería el eje responsable de la dinámica rotacional del universo observable tridimensional.

Por tanto, se llega a la siguiente conclusión: para que el universo sea dinámico requiere que sea un sistema abierto con dimensiones espaciales complementarias consecutivas y debe existir un eje de rotación en una dimensión espacial extra. Teniendo en cuenta el concepto general de superficie esférica podemos construir diagramas simples, los cuales aclaran los conceptos del universo abierto y de las dimensiones espaciales complementarias.

El universo abierto de dimensión cero (0) puede describirse mediante una superficie esférica de dimensión cero, como se muestra en la figura 5. Supongamos que a cada sistema natural le corresponde una abertura, entonces el universo abierto de dimensión cero estaría conformado por dos aberturas, siendo una de ellas la imagen especular de la otra. Obsérvese que el centro de la superficie esférica de dimensión cero (0) se encuentra en el espacio de dimensión uno (1). Un ser de este universo no se daría cuenta con su percepción sensorial que en realidad vive en $0+$ $1=1$ dimensión. Su mundo trascendente y objetivo es una esfera de dimensión uno cuyo centro sería su sistema de referencia inercial. La doble flecha proveniente del centro indica la dualidad intrínseca asociada a las aberturas objeto/imagen (extremos), las cuales conforman una sola entidad. Nótese que cualquier eje perpendicular a la flecha dual que pase por su centro engendrará un universo dinámico animado con rotación, traslación y vibración.

El universo abierto de dimensión uno (1) se describe como el lugar geométrico de puntos situados sobre una superficie esférica unidimensional (una circunferencia), cada uno de ellos con su imagen especular respectiva, como muestra la figura 6 . Su centro se encuentra en un punto del espacio bidimensional (el centro del círculo). Un ser de este universo no se daría cuenta con su percepción sensorial que en realidad vive en $1+2=3$ dimensiones. Su mundo trascendente y objetivo es una esfera de dimensión dos (2) cuyo centro sería su sistema de referencia inercial. Por tanto, para que el universo unidimensional sea abierto y dinámico, la dimensión uno (1) debe complementarse con la dimensión dos (2) y debe existir un eje de rotación inercial situado en la dimensión tres (3), el cual es perpendicular a cada flecha dual en su centro.

El universo abierto de dimensión dos (2) se describe como el lugar geométrico de puntos situados sobre una superficie esférica bidimensional, cada uno de ellos con su respectiva imagen especular, como muestra la figura 7 . Las flechas dobles indican ejes de rotación localizados en la dimensión tres, los cuales pasan a través de las aberturas y generarían las dos dimensiones o ejes espaciales. El centro de este universo se encuentraría en un punto del espacio tridimensional (el centro de la esfera). Un ser bidimensional no podría percibir que vive en $2+3=5$ dimensiones. Su mundo trascendente y objetivo es una esfera de dimensión tres (3) cuyo centro sería su sistema de referencia inercial. El eje de rotación inercial de este universo se encuentra en la dimensión cuatro (4). Por tanto, para que el universo bidimensional sea abierto y dinámico, la dimensión 2 debe complementarse con la dimensión 3 y debe existir un eje de rotación inercial situado en la dimensión 4, el cual no se percibe. En analogía con el universo unidimensional, el universo bidimensional junto con su complemento, el universo tridimensional, estarán sometidos a una rotación rígida alrededor de este eje inercial.

$Y$ se llega al caso más interesante: el universo abierto de dimensión tres (3). Se describe como el lugar geométrico de puntos localizados sobre una superficie esférica tridimensional, cada uno de ellos con su respectiva imagen especular. Su centro se encuentra en un lugar del espacio 
tetradimensional. Pero, ¿cómo es ese lugar? Podríamos esperar que la simetría cónica, intrínseca al espacio tridimensional, continúe en el espacio tetradimensional; pero no podemos imaginar su aspecto. Sin embargo, podemos continuar utilizando nuestro elemento dual (la doble flecha) el cual debe pasar por el centro de la inimaginable esfera tetradimensional, como se muestra en la figura 8. En este caso las flechas dobles indican ejes de rotación localizados en la dimensión cuatro los cuales pasarían por las posibles aberturas (probablemente incandescentes) que existirían los sistemas naturales autoorganizados, autónomos con funcionamiento inteligente del espacio tridimensional, y generarían las tres dimensiones espaciales o ejes que nuestra mente percibe. Seres tridimensionales, como nosotros los humanos, no podríamos percibir con nuestros órganos sensoriales que vivimos en $3+4=7$ dimensiones.

Por consiguiente, el mundo trascendente inmediato es la esfera de dimensión cuatro (4), siendo su centro el lugar donde se deberá ubicar nuestro sistema de referencia inercial respecto al cual se describiría la realidad objetiva. Cada abertura del universo tridimensional observable (correspondiente a un sistema natural) se hallaría sostenida, revitalizada y dirigida a cada instante por influencias las cuales se localizarían en el interior de la esfera tetradimensional. Estas influencias constituirían un programa, el cual sería responsable de los fenómenos naturales que observamos. El eje de rotación inercial de la superficie tridimensional que percibimos y de su complemento, el espacio tetradimensional, se encuentra en la dimensión cinco (5). Por tanto, para que el universo tridimensional sea abierto y dinámico la dimensión 3 debe complementarse con la dimensión 4 y debe existir un eje de rotación inercial situado en la dimensión 5 que pasa por la abertura correspondiente al universo observable: el centro de la esfera tetradimensional, el cual sería nuestro sistema de referencia inercial en primera aproximación.

Para que existan influencias procedentes del espacio de dimensión 4, las cuales produzcan los efectos que percibimos en el espacio de dimensión 3 (como fenómenos naturales), un eje de rotación localizado en la dimensión 5 debe generar infinitos ejes de rotación los cuales se localicen en el espacio de dimensión 4, y constituyan las influencias externas al espacio de dimensión 3. Estos ejes pueden considerarse inerciales, en primera aproximación, respecto al espacio tridimensional. A su vez podrían desdoblarse en otros ejes de rotación localizándose en el espacio tridimensional que podemos percibir con nuestros sentidos; pero estos ejes no pueden admitirse como inerciales porque están conectados a los ejes de rotación externos.

\section{Conclusiones}

De acuerdo con el pensamiento sistémico, cuya base filosófica es el holismo, y teniendo en cuenta la filosofía del funcionalismo, el universo debe considerarse como sistema abierto, como si fuese un organismo vivo con mente, dinámico y con simetría esférica.

La perspectiva de un sistema de referencia inercial sería, en primera aproximación, el foco de lectura de la mente humana y se hallaría en el centro de una inimaginable esfera tetradimensional. En este lugar confluirían, por algún mecanismo, las líneas de visión humana para que nuestra visión del universo a gran escala sea la misma en cualquier dirección y desde cualquier posición.

En el contexto de la hipótesis del universo abierto la interpretación de los experimentos de la inversión especular y del espín del electrón implican que los sistemas naturales autoorganizados, autónomos y con funcionamiento inteligente del espacio tridimensional estén conectados de algún modo a un eje de rotación situado en una dimensión espacial extra. De lo contrario no se podrían explicar con sencillez las rotaciones, y los subsecuentes fenómenos naturales (debido a la dinámica del universo) con respecto a un sistema de referencia inercial (en primera aproximación).

Como consecuencia de interpretar los experimentos de la inversión especular y del espín del electrón, se puede afirmar que el universo observable sería un sistema dual en el sentido de que tendría dos superficies tridimensionales complementarias opuestas con simetría especular las cuales conforman una sola entidad. Esta conclusión se podría fundamentar y generalizar con el siguiente postulado: la superficie opuesta de un objeto de dimensión $n$ es su imagen en un espejo de dimensión $n \backsim 1$, siempre que las normales al espejo desde cada punto del objeto y desde cada punto de su imagen son opuestas entre si.

Para definir una hiperesfera las dimensiones espaciales deben complementarse de modo consecutivo. Esto se puede sustentar y generalizar a partir de la siguiente proposición lógica, que podemos denominar postulado de las dimensiones espaciales complementarias: toda hiperesfera está conformada por una hipersuperficie de dimensión $n$ que encierra un hipervolumen de dimensión $n+1$, ( $\mathrm{n}=$ $0,1,2,3, \ldots)$.

Basándose en el postulado de las dimensiones espaciales complementarias es posible construir diagramas simples para explicar la idea del universo abierto y dinámico mediante la siguiente regla: si en una hiperesfera la hipersuperficie de dimensión $n$ se complementa con el hipervolumen de dimensión $n+1$, entonces siempre existirá un eje de rotación inercial en la dimensión $n+2$ que pase por el centro de la hiperesfera. Por tanto, llegamos a la conclusión de que no existe sistema de referencia inercial absoluto, sino solamente aproximaciones sucesivas. Si un sistema de referencia se considera inercial en un espacio de dimensión $n$, no lo será en un espacio de dimensión $n+1$.

El postulado de las dimensiones espaciales complementarias implica, en general, que la ejecución del programa 
ineherente a la mente de un sistema en un espacio de dimensión $n$ consiste en dibujar en cada instante los acon- tecimientos en el espacio de dimensión $n-1$, lo cual constituyen sus efectos.

\section{Referencias}

[1] A. Einstein y L. Infeld. La evolución de la Física, Salvat Editores S.A, Barcelona, España. (1986).

[2] O. Monroy. La escala de Planck, Revista de Investigación de Física, Vol. 12, N 1, Perú. (2009).

[3] H. Bondi. Cosmología, Editorial Labor, S.A, Barcelona, España. (1970).

[4] S. Hawking. Historia del tiempo, Serie Mayor. Grupo Editorial Grijalvo, S.A, Bogotá, Colombia. (1989).

[5] P. Davies. Dios y la nueva Física, Salvat Editores, España. (1986).
[6] M. Gardner. Izquierda y derecha en el cosmos, Salvat editores S.A. Barcelona, España. (1985).

[7] E. Abbot. Flatland: A romance of many dimensions, Princeton University Press, USA. (1991).

[8] B. Greene. El universo elegante, Editorial Planeta colombiana S.A, Barcelona, España. (2007).

[9] R. Penrose. La mente nueva del emperador. (En torno a la cibernética, la mente y las leyes de la Física, Fondo de Cultura Económica, México. (1996).

[10] R. Penrose. El camino a la realidad, Debate, México. (2007). 